\title{
Distribución geográfica de la avifauna y la ciencia ciudadana en el País Vasco: una aproximación a través del portal www.ornitho.eus
}

\section{Geographical distribution of birds and citizen science in the Basque Country: an approach from the www.ornitho.eus portal}

Juan Arizaga ${ }^{1 *}$, Javier Rodríguez-Pérez ${ }^{1}$

*

\section{Resumen}

El objetivo de este artículo es analizar la distribución geográfica de las observaciones que se registran en la plataforma www.ornitho.eus, con el fin de describir su patrón de distribución espacial, detectar zonas tanto de alta como de baja densidad de observaciones y establecer directrices para la mejora del esfuerzo de muestreo en el territorio. Para ello se han empleado las observaciones de aves registradas durante un periodo de un año una vez la plataforma se puso en funcionamiento en marzo de 2015 (para los análisis han sido considerados los datos que se obtuvieron entre los meses de junio de 2015 a mayo de 2016). Se obtuvieron un total de 94.222 observaciones: 49.000 (52\%) observaciones se produjeron en Gipuzkoa, seguidas de 30.213 (32\%) en Bizkaia y 15.009 (16\%) en Álava. En términos absolutos hallamos varias zonas con altas densidades de citas: el NE de Gipuzkoa (esto es, el entorno de Txingudi), Donostialdea, Urdaibai, el gran Bilbao, el SO de Gipuzkoa (el entorno de Oñati), la ciudad de Vitoria-Gasteiz, embalses alaveses de Ullibarri-Ganboa y el entorno de Laguardia. Por otro lado, las zonas sin o con menos citas fueron, principalmente, puntos en el interior de Gipuzkoa y Bizkaia y el SO de Álava. Se registró una asociación positiva entre el número de citas en cada cuadrícula y el porcentaje de cobertura de humedales y área urbanizada en la misma.

Palabras clave: Observadores de aves, paisaje, portales de internet de biodiversidad, voluntariado.

\footnotetext{
${ }^{1}$ Departamento de Ornitología, Sociedad de Ciencias Aranzadi.

Zorroagagaina 11, 20014 Donostia.
} 


\begin{abstract}
The aim of this article is to analyze the geographic distribution of sightingings recorded on the www.ornitho.eus internet portal, with the aim of describing their spatial distribution pattern, detecting areas of both high and low density of records and establishing guidelines to improve sampling efforts within the region covered by this portal. For this purose we used bird data collected over a period of one year, once the portal had begun to operate in March 2015 (the data used in the paper are those collected from June of 2015 to May of 2016). Overall, 94,222 records were obtained: 49,000 (52\%) in Gipuzkoa, 30,213 (32\%) in Bizkaia and 15,009 (16\%) in Álava. Those areas with a high density of records were: NE Gipuzkoa (i.e., Txingudi area), Donostialdea, Urdaibai, Bilbao metropolitan area, SW Gipuzkoa (around Oñati municipality), VitoriaGasteiz, Ullibarri-Ganboa reservoirs and the area round Laguardia. Those zones with a lower density of records were a number of areas from Gipuzkoa and Bizkaia and SW Álava. We detected a positive association between the density of records and the percentage of cover of wetlands and urban areas within a grid.
\end{abstract}

Key words: Birdwatchers, landscape, biodiversity internet portal, volunteers.

\title{
Laburpena
}

Artikulu honen helburua www.ornitho.eus plataforman erregistratuta dauden behaketen banaketa geografikoa aztertzea da, zertarako eta horien banaketa espazialaren patroia deskribatzeko, behaketa dentsitate altuko zein baxuko eremuak antzemateko eta lurraldean egiten den laginketa lana optimizatzeko gidalerroak ezartzeko. Horretarako 2015. urteko martxoan plataforma abian jarri ondoren urtebeteko epean bildutako hegazti behaketak erabili dira (analisietarako 2015eko ekainetik 2016ko maiatzera bitarte lortutako datuak hartu dira). Guztira 94.222 behaketa egin ziren: horietatik 49.000 (\%42) Gipuzkoan jaso ziren, Bizkaian jasotakoak 30.213 (\%32) izan ziren eta Araban 15.009 (\%16). Datu absolutuetara ekarrita, aipamen dentsitate altuko hainbat eremu ageri dira: Gipuzkoa IE (hau da Txingudi ingurua), Donostialdea, Urdaibai, Bilbo Handia, Gipuzkoa HM (Oñati ingurua), Vitoria-Gasteiz hiria, Ulibarri-Ganboa urtegiak eta Guardia ingurua. Bestalde, aipamen gutxirekin edo bat ere gabe agertutako eremu aipagarrienak Gipuzkoako eta Bizkaiko barnealdeko gune batzuk izan dira eta Araba HM eremua. Eta elkarreragin positiboa ere ageri da: lauki sare bakoitzeko aipamen kopuruan eta hezeguneen estaldura portzentajean eta horien azalera urbanizatuaren artean.

Gako hitzak: hegazti behatzaileak, paisaia, Interneteko biodibertsitate atariak, boluntarioak. 


\section{Introducción}

En el ámbito de la biogeografía, la distribución y fenología de especies, el aporte de datos desde la ciudadanía (esto es, voluntariado) se está consolidando como una herramienta muy útil para la conservación de la biodiversidad y el desarrollo de las ciencias ecológicas (Bonney et al., 2009; Dickinson et al., 2012; Jiguet et al., 2012; Tulloch et al., 2013). Aunque es evidente que la recopilación sistemática de información, a través de protocolos o experimentos bien planificados, proporciona la base para determinar relaciones causa-efecto en estudios de biodiversidad, a menudo y para muchas especies la recolección de cantidades importantes de información no es factible ni espacial ni temporalmente (Kelling et al., 2015). En este contexto, los datos que se obtienen a partir de la aportación de la ciudadanía tienen un cada vez más alto valor tanto en el ámbito de la ciencia como en el de la conservación de la biodiversidad, pues permiten abarcar amplias escalas temporales y geográficas (Devictor et al., 2010). Además, los portales de internet destinados a recolectar datos a partir de la ciencia ciudadana están facilitando el establecimiento de puntos de encuentro y sinergias entre comunidad científica y ciudadanía a la par que permiten la obtención de datos y patrones de manera rápida (Dickinson et al., 2012). Aun así, el patrón de las observaciones y esfuerzo de muestreo en este tipo de plataformas es heterogéneo, por lo que la distribución geográfica de citas se asocia a la distribución geográfica de observadores, entre otros sesgos (Isaac et al., 2014; Lukyanenko et al., 2016). En este contexto, y con el fin de mejorar la calidad de los datos que se obtienen a partir de este tipo de fuentes, es importante determinar el patrón de distribución espacial de dichas observaciones.

Existe actualmente un auge de las plataformas de ciencia ciudadana orientadas a la recopilación masiva de datos sobre la distribución de fauna (Amano et al., 2016). En el caso particular de la avifauna, son varias las existentes a escala global y en Europa en particular. En los países que conforman la UE, algunas de las más utilizadas son: ornitho, ebird, artportalen, artsobservasjoner, aves-symfony, birdtrack, trektellen, observation, vogelatlas... (para más detalles ver www.eurobirdportal.org). La Comunidad Autónoma del País Vasco (CAPV) no ha sido ajena a este proceso, ya que a partir de marzo de 2015 se implementó, de la mano de la Sociedad de Ciencias Aranzadi, y en colaboración con el Gobierno Vasco, la plataforma www.ornitho.eus. Esta plataforma se diseñó para la recopilación de citas de fauna, donde el grupo con más citas es, indiscutiblemente, el de las aves. Con fecha de 31.12.2015, el $94,8 \%$ de las citas correspondía a observaciones de aves (Arizaga, 2015). Así, la plataforma www.ornitho.eus ha supuesto un avance significativo en la recolección de datos sobre la avifauna de la CAPV. Sólo en 2015 se registraron > 60.000 observaciones, lo cual constituye un hito en la historia de la ornitología en el territorio.

El objetivo de este artículo es analizar la distribución geográfica de las observaciones que se registran en la plataforma www.ornitho.eus, con el fin de describir su patrón geográfico, detectar zonas tanto de alta como de baja densidad de observaciones y establecer directrices para la mejora del esfuerzo de muestreo en el territorio. Es esperable que las zonas más accesibles o cercanas a entornos poblados o en los que haya alta diversidad de especies (tales como zonas húmedas y espacios protegidos) contengan proporcionalmente un número de citas mayor al de su entorno más próximo. 


\section{Material y métodos}

\section{Entorno geográfico, recopilación y procesamiento de datos}

El área geográfica del presente estudio corresponde a la CAPV, una región que abarca 7.234 km². La CAPV cuenta con cuatro regiones climáticas. Así, la región o vertiente atlántica comprende la totalidad de las provincias de Gipuzkoa y Bizkaia y parte del norte de Álava. El clima en esta región, mesotérmico, es moderado en cuanto a temperaturas y lluvioso. Posteriormente se extienden, abarcando casi toda la provincia de Álava, la región subatlántica y submediterránea, con climas más secos y menos lluviosos. Finalmente, en el sur de Álava el clima es ya totalmente mediterráneo, con veranos muy secos y calurosos e inviernos fríos y poco lluviosos.

El proyecto ornitho nació en Suiza, como medio para recopilar citas sobre aves en este país. En la actualidad, el proyecto se ha implantado, a través de portales personalizados a nivel local, en varios países o regiones de Europa. El sistema ornitho, y el portal www.ornitho.eus en particular, está adaptado para el registro de citas tanto casuales como a través de lo que se denomina "listas completas". Tales listas incluyen un registro sistematizado de todas las especies que se han visto en un lugar y lapso de tiempo determinados. Por otro lado, el portal está siendo habilitado para recopilar citas recogidas a través de protocolos de censo, tales como pueden ser los censos invernales de aves acuáticas, censos de aves coloniales o, en general, cualquier protocolo de muestreo que requiera la recopilación de información adicional o la aplicación de metodologías de censo que vayan más allá del formulario estándar de recogida de citas.

Para llevar a cabo este estudio se consideraron todas las citas de aves registradas en www.ornitho.eus para el periodo 01.06.2015 a 31.05.2016. La descarga de datos se realizó el 15.06.2016. Para ese periodo son registradas un promedio de 7248 observaciones por mes (la media en 2015 es 5.878 y, en 2016, 9.440). En conjunto, se recopiló un total de 94.222 observaciones. Cada cita contiene, como mínimo, la siguiente información: fecha, taxón (normalmente, especie), posición geográfica (con una resolución espacial mínima de $1 \mathrm{~km}^{2}$ ). Posteriormente, todas las citas se agruparon en cuadrículas de $5 \times 5 \mathrm{~km}$ de resolución, como compromiso entre una malla de resolución fina para describir y detectar patrones geográficos a escala regional (en este caso, la CAPV), pero lo suficientemente grande para evitar un alto número de cuadrículas sin información.

Para analizar la relación entre la distribución de citas y el paisaje se obtuvieron datos de cobertura paisajística a partir de CORINE Land Cover 2006 para la CAPV a una resolución de 250×250 m. El mapa original consta de 39 categorías de clases de cobertura de terreno. En cada cuadrícula de 5×5 km, esta información se empleó para calcular el porcentaje de cobertura de humedales (superficies de agua tanto dulce como salobre o salina; incluye lagunas, marismas, embalses, ríos...) y hábitats urbanizados (núcleos urbanos, industrias, parques urbanos). Adicionalmente, a través del portal de GeoEuskadi, se calculó en cada cuadrícula de $5 \times 5 \mathrm{~km}$ el porcentaje de superficie de LICs (Lugares de Importancia Comu- 
nitaria) y el número de habitantes. Para el manejo y procesamiento de datos se utilizaron los paquetes "rgdal" (v. 1.1-10), "raster" (v. 2.5-2), "maptools" (v. 0.8-39) bajo el lenguaje de programación R v. 3.2.3 (R Core Team, 2014)

\section{Análisis de datos}

Para describir la distribución geográfica de las observaciones así como su asociación con potenciales variables predictivas, se usaron métodos de geo-estadística cuyo objetivo es la identificación y descripción de patrones espaciales y el testeo de hipótesis con un componente espacial explícito (Turner et al., 2001). Los análisis se realizaron con los paquetes "sp" (v. 1.2-3), "spdep" (v. 0.6-6) y "lattice" (v. 0.20-33) en R v. 3.2.3 (R Core Team, 2014).

Primeramente, se representó la densidad de observaciones para la CAPV, en escala logarítmica. Esta transformación se aplicó debido al alto número de observaciones acumuladas en el área de Txingudi (NE de Gipuzkoa).

Posteriormente, para determinar si hubo autocorrelación espacial en el número de observaciones de avifauna se aplicó el test de la I de Moran. El estadístico radopta un valor entre -1 y +1 . Con este método determinamos si las citas están distribuidas de manera agregada $(r>0)$, aleatoria $(r \sim 0)$ o disgregada $(r<0)$ para diferentes intervalos de distancia (Fortin \& Dale, 2005).

A continuación, se caracterizó el patrón geográfico de las observaciones en relación a las citas del entorno más inmediato a partir de mapas de autocorrelación espacial local. El análisis LISA (Local Indicators of Spatial Association), basado en estadísticos locales de la I de Moran Local, permite medir el nivel de similitud en la densidad de citas entre celdas próximas respecto de lo que cabría hallar por azar y, así, identificar cuadrículas de alta o baja densidad local (Dale et al., 2002).

Finalmente, analizamos la asociación entre las citas de avifauna y variables de paisaje que pudieran explicar la distribución geográfica de tales citas. Aplicamos con este fin modelos Auto-Regresivos Simultáneos (SAR), en los que se asume dependencia (lineal) espacial entre los datos (Fortin \& Dale, 2005). Así, se aplicaron modelos lineales ( $\mathrm{g} / \mathrm{m}$ ) para predecir la relación entre el número de citas y covariables: el porcentaje de cobertura de terreno ocupada por humedales o núcleos urbanos, el porcentaje de superficie LIC y el tamaño de la población humana. Para determinar qué modelo fue el que se ajustó mejor a los datos se evaluó mediante el Criterio de Información de Akaike (AIC) (Burnham \& Anderson, 1998)

\section{Resultados}

Para el periodo de estudio se obtuvieron un total de 94.222 observaciones. Por provincias, 49.000 (52\%) observaciones se produjeron en Gipuzkoa, seguidas de 30.213 (32\%) en Bizkaia y 15.009 (16\%) en Álava. 


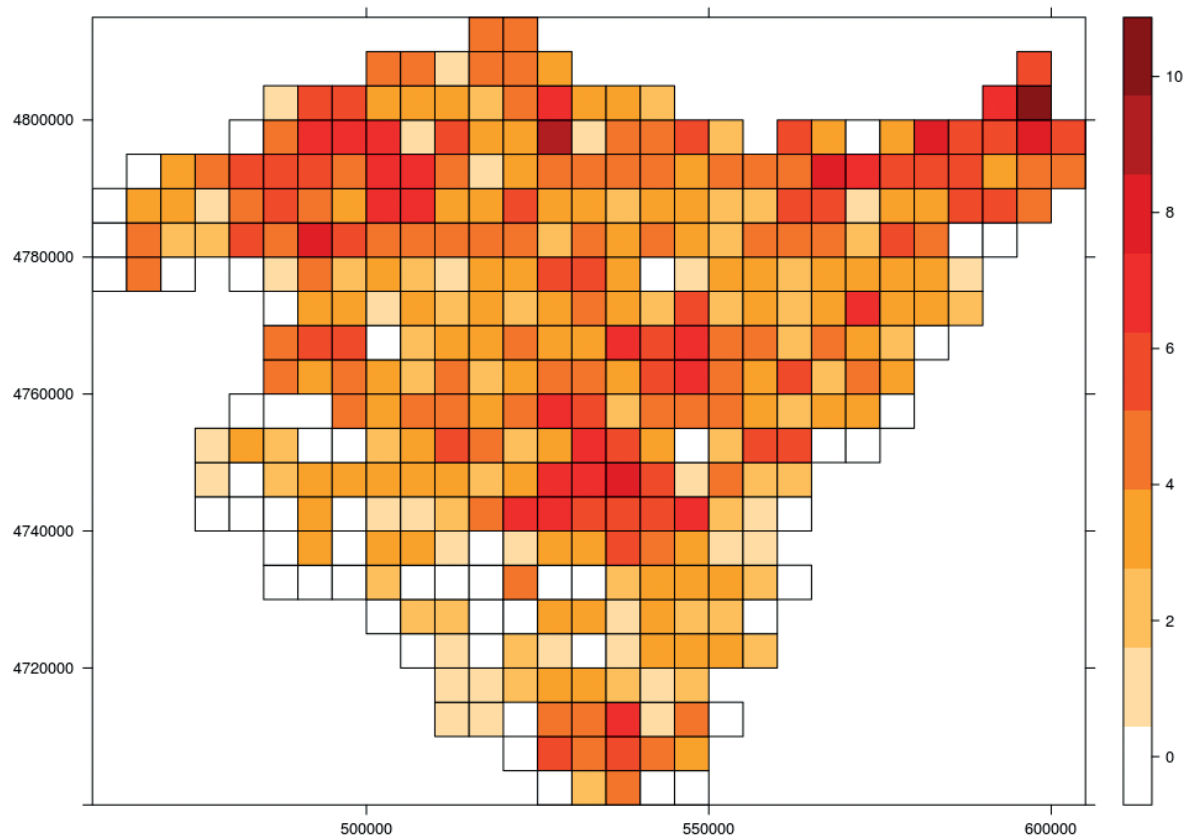

Fig. 1.- Distribución geográfica (datos en bruto, a escala log) de las observaciones de aves en la CAPV, durante el periodo 01.06.2015-31.05.2016. El número de observaciones se ha agrupado según una malla de cuadrícula de $5 \times 5 \mathrm{~km}$. La coloración de la cuadrícula indica la densidad de observaciones (alta densidad, en tonos rojos; baja densidad, en amarillo y blanco).

Fig. 1.- Geographic distribution (log-scaled raw data) of bird record data within the Basque region, during the period 01.06.2015-31.05.2016. Data have been shown using a $5 \times 5 \mathrm{~km}$ grid. The colors represent the densities of bird records (high densities have been represented in red and low densities, in yellow or white.

Hallamos varias zonas con altas densidades de citas (de E a O y de N a S; Fig. 1): el NE de Gipuzkoa (esto es, el entorno de Txingudi), Donostialdea, Urdaibai, el gran Bilbao, el SO de Gipuzkoa (el entorno de Oñati), la ciudad de Vitoria-Gasteiz y embalses alaveses de Ullibarri-Ganboa, el entorno de Laguardia. Por otro lado, las zonas sin o con menos citas son, principalmente, determinados puntos en el interior de Gipuzkoa y Bizkaia y el SO de Álava.

En cuanto al patrón geográfico, éste es, claramente, agregado, especialmente en distancias de 5 a 35 km (Fig. 2). Esto es, la mayoría de observaciones se halló en zonas próximas entre sí hasta una distancia de c. 35 km, lo que indica que existe agregación de observaciones para el conjunto de la CAPV. Entre 35 y $80 \mathrm{~km}$ el patrón es aleatorio y, a distancias $>85 \mathrm{~km}$ se presenta un patrón de repulsión, tal vez debido a la existencia de zonas de contraste entre amplias zonas de alta y baja densidad de observaciones.

El análisis LISA para detectar zonas de alta y baja densidad local en el número de observaciones muestra núcleos de agregación en torno a las capitales, así como, también, en otras 


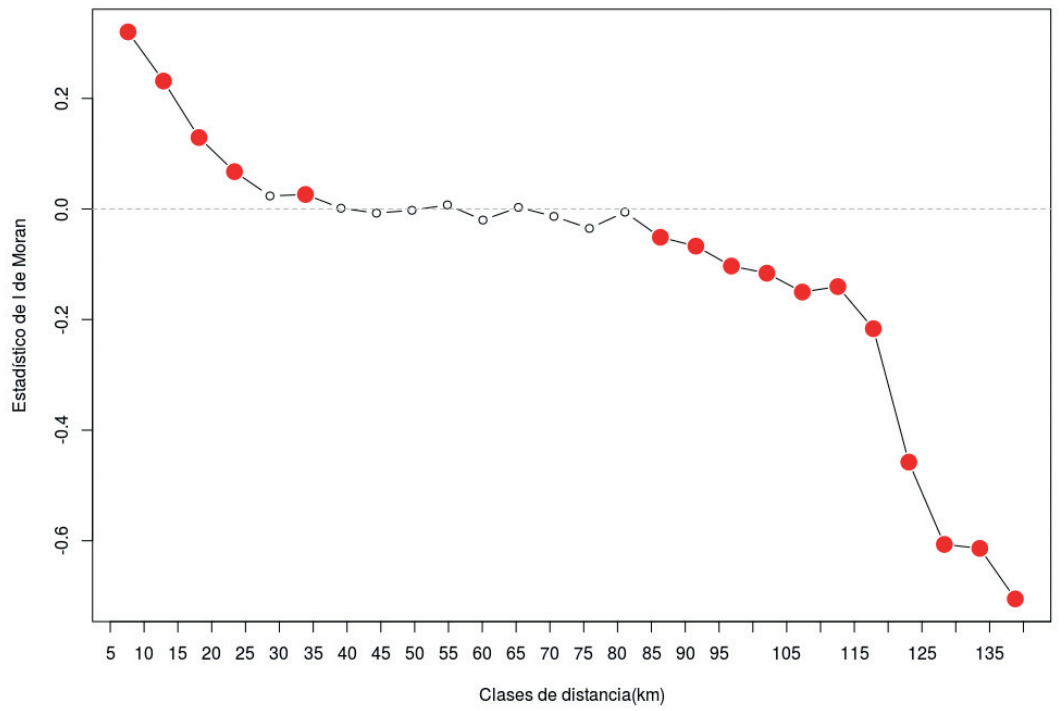

Fig. 2.- Polígono mínimo convexo de cuatro ejemplares adultos de gaviota patiamarilla marcados en una de las colonias de cría en Gipuzkoa (Ulía: punto verde), durante el periodo 24.05.2016-31.07.2016. En marrón se representan los dos vertederos utilizados por las gaviotas marcadas (Sasieta y Zaluaga).

Fig. 2.- Minimum convex polygons relative to four adult yellow-legged gulls from the Ulia colony (green point), during the period 24.05.2016-31.07.2016. Brownish points show the two open dumps used by these four gulls (Sasieta and Zaluaga).

zonas, tales como Txingudi, Urdaibai o el SO de Álava (Fig. 3). A diferencia de la Fig. 1, los resultados que observamos en la Fig. 3 no necesariamente muestran alta densidad absoluta de observaciones; en determinadas zonas puede haber alta densidad local de observaciones, sobre todo si en las cuadrículas más próximas el número de observaciones es nulo o muy bajo. La Fig. 3, en definitiva, muestra máximos locales, esto es, puntos o zonas de concentración de observaciones en relación a las áreas más próximas a tales zonas, donde habría pocas.

Los resultados de los modelos SAR indican que el porcentaje de superficie de humedales, de área urbana y de superficie de LICs tuvo una relación positiva con el número de observaciones de aves (Tabla 1). No obstante, el valor de lambda $(\lambda=0,5492, P<0,001)$, significativamente alto, indica autocorrelación de residuales, lo que sugiere que el modelo no es capaz de capturar toda la variabilidad geográfica asociada a la distribución de observaciones y, en consecuencia, deben existir otras covariables a la hora de explicar la distribución de las observaciones. Modelos SAR posteriores, ponderados para la densidad de población humana (esto es, esperaríamos más citas donde hay más observadores), todavía indican un efecto positivo de la proporción de superficie de área urbana en el número de citas, 


\begin{tabular}{|lccc|}
\hline Covariable & B & SE (B) & P \\
\hline A: log(observaciones) & HUM+URB+LIC; AIC $=1473,4$ & 0,030 \\
\hline HUM & $+0,083$ & 0,034 & $<0,001$ \\
URB & $+0,065$ & 0,014 & 0,006 \\
LIC & $+0,013$ & 0,005 & \\
\hline B: log(observaciones) $\sim$ HUM + +URB+LIC, ponderado para POB; AIC = 2519,4 & 0,644 \\
\hline HUM & $+0,008$ & 0,075 & $<0,001$ \\
URB & $+0,048$ & 0,004 & 0,464 \\
LIC & $+0,007$ & 0,009 & \\
\hline
\end{tabular}

Tabla 1.- Coeficientes ( $B$-parámetros) obtenidos para el primer modelo SAR desarrollado para determinar el efecto de varias covariables en la densidad de observaciones de aves en la CAPV, tanto no (A) como sí (B) ponderado para el número de habitantes en la cuadrícula. Abreviaciones: HUM, porcentaje de humedales en la cuadrícula de $5 \times 5 \mathrm{~km}$; URB, porcentaje de área urbanizada; LIC, porcentaje de espacios declarados LIC; POB, número de habitantes en la cuadrícula; AIC, criterio de información de Akaike.

Table 1.- Coefficients ( $B$-parameters) obtained from the first SAR model used to test for the effect of a number of covariates on bird record densities across the Basque region, either removing $(A)$ or considering (B) the number of inhabitants within each square. Abbreviations: HUM, percentage of wetlands within each $5 \times 5 \mathrm{~km}$ square; URB, percentage of urban areas LIC, percentage of LIC (Sites of European Interest); $\mathrm{POB}$, number of inhabitants with each square; AIC, Akaike information criterion.

pero no así de la superficie de LIC ni de humedales. A pesar de que el valor de lambda continúa siendo significativamente alto, se reduce a la mitad con respecto al primer análisis sin ponderar por la población humana $(\lambda=0,2688, P=0,001)$.

\section{Discusión}

Basándonos en el portal www.ornitho.eus, inaugurado en marzo de 2015, la distribución de observaciones de aves en la CAPV está notoriamente agregada. En este contexto, la cobertura de observación en Álava es, netamente, inferior a la de las otras dos provincias. Así, el número de citas en esta provincia sólo supone un 16\% del total de citas para la CAPV.

La Fig. 3 revela una franja geográfica de alta densidad local en el valle del río Ebro, en particular en los límites del SO de Álava. Al examinar la distribución absoluta de citas (Fig. 1), no obstante, observamos un alto número de cuadrículas sin o con muy pocas citas en esta zona. En consecuencia, el escaso número de cuadrículas con citas son detectadas como puntos de alta densidad local cuando vemos los resultados del análisis LISA. Se pone de manifiesto, así, que el SO de Álava está muy poco cubierto y es, en consecuencia, una de las zonas donde convendría aumentar el esfuerzo de muestreo. 


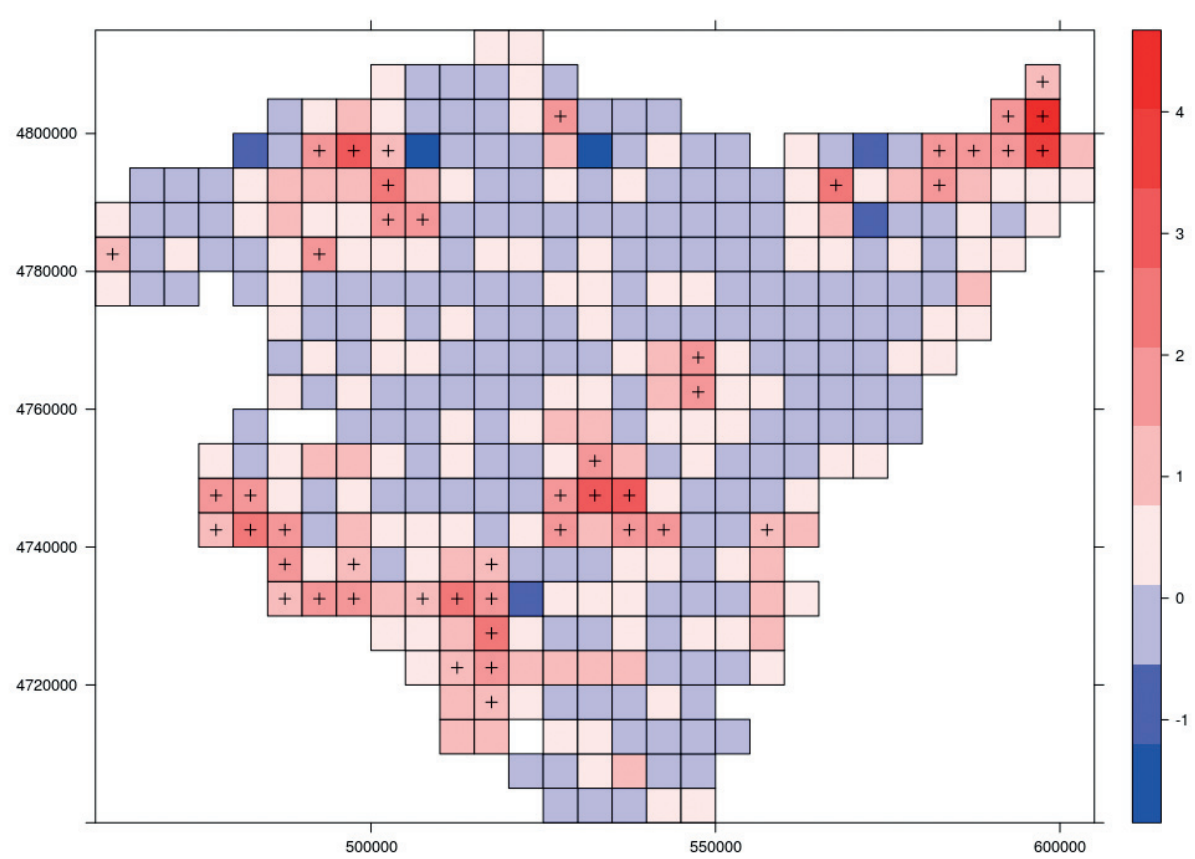

Fig.3.- Distribución de los valores de la I de Moran Local del número de observaciones de avifauna. Los estadísticos de correlación local indican si el número de observaciones en la cuadrícula es significativamente superior (+) a los valores que se observan en las cuadrículas del entorno más inmediato.

Fig. 3.- Distribution of the I Local Moran values. These statistics show whether the number of records in a square is significantly higher (+) to the values observed in nearby squares.

La distribución de observaciones de aves en la CAPV a través del portal www.ornitho.eus se aglutina fundamentalmente en torno al área urbanizada de cada cuadrícula. Esta tendencia queda aún mejor reflejada al controlar el número de citas en cada cuadrícula por el número de habitantes. Por otro lado, se registra una asociación positiva entre el número de citas y el porcentaje de cobertura de humedales. Este patrón responde al hecho de que, por un lado, los humedales son un polo de atracción de observadores, dada la diversidad de especies y la facilidad de observación, especialmente en zonas donde se han habilitado infraestructuras para ello. El alto número de citas tanto en Txingudi como en Urdaibai, los estuarios más importantes de la costa vasca, explicaría la alta asociación entre el número de citas y el porcentaje de marismas en la cuadrícula. Aun así, el porcentaje de área debida a espacios LIC no se asocia al número de citas de aves, al menos cuando ponderamos para el número de habitantes. Esto lleva a la conclusión de que la cobertura en la red Natura 2000 no es particularmente alta (salvo en zonas húmedas), siendo la heterogeneidad en el esfuerzo de muestreo uno de los problemas más importantes a los que se enfrentan los 
portales de ciencia ciudadana (Isaac et al., 2014; Amano et al., 2016). En la CAPV, los modelos son capaces de explicar, al menos en parte, las causas que determinan el patrón de distribución geográfica de observaciones de aves en el territorio.

En conclusión, existe para la CAPV un esfuerzo de muestreo desigual, con una alta concentración de citas en humedales (e.g., Txingudi, Urdaibai, Salburua), una cobertura aceptable (aunque con puntos sin cubrir) en Gipuzkoa y Bizkaia y una cobertura más deficiente en Álava, especialmente en el SO de la provincia. En este escenario sería necesario llevar a cabo un programa para formar e incentivar la participación de observadores en aquellas zonas con menos cobertura. Se podrían también aplicar iniciativas alternativas, como incentivar la visita a estas zonas menos pobladas por parte del colectivo de aficionados a las aves del territorio, o financiar desde los entes públicos el muestreo de aves a través de profesionales (guardas, etc.).

\section{Agradecimientos}

El proyecto www.ornitho.eus es posible gracias al apoyo del Gobierno Vasco, Diputaciones vascas, diversas asociaciones y organismos públicos y privados y, ante todo, la contribución de cientos de observadores y un comité de expertos para la verificación de citas. M. Díaz y un revisor anónimo aportaron algunos comentarios que contribuyeron a mejorar una primera versión del trabajo.

\section{Bibliografía}

- Amano, T., Lamming, J.D.L., Sutherland, W.J. 2016. Spatial Gaps in Global Biodiversity Information and the Role of Citizen Science. BioScience 66: 393-400.

- Arizaga, J. 2015. Plataforma www.ornitho.eus. Balance del año 2015. Inédito. Sociedad de Ciencias Aranzadi. Donostia.

- Bonney, R., Cooper, C.B., Dickinson, J., Kelling, S., Phillips, T., Rosenberg, K.V., Shirk, J. 2009. Citizen Science: A Developing Tool for Expanding Science Knowledge and Scientific Literacy. BioScience 59: 977-984.

- Burnham, K.P., Anderson, D.R. 1998. Model Selection and Inference. A Practical Information Theoretic Approach. Springer-Verlag. New York.

- Dale, M.R.T., Dixon, P., Fortin, M.-J., Legendre, P., Myers, D.E., Rosenberg, M.S. 2002. Conceptual and mathematical relationships among methods for spatial analysis. Ecography 25: 558-577.

- Devictor, V., Whittaker, R.J., Beltrame, C. 2010. Beyond scarcity: citizen science programmes as useful tools for conservation biogeography. Diversity and Distributions 16: 354-362. 
- Dickinson, J.L., Shirk, J., Bonter, D., Bonney, R., Crain, R.L., Martin, J., Phillips, T., Purcell, K. 2012. The current state of citizen science as a tool for ecological research and public engagement. Frontiers in Ecology and the Environment 10: 291-297.

- Fortin, M.J., Dale, M.R. 2005. Spatial analysis: a guide for ecologists. Cambridge University Press. Cambridge.

- Isaac, N.J.B., Van Strien, A.J., August, T.A., de Zeeuw, M.P., Roy, D.B. 2014. Statistics for citizen science: extracting signals of change from noisy ecological data. Methods in Ecology and Evolution 5: 1052-1060.

- Jiguet, F., Devictor, V., Julliard, R., Couvet, D. 2012. French citizens monitoring ordinary birds provide tools for conservation and ecological sciences. Acta Oecologica 44: 58-66.

- Kelling, S., Fink, D., Sorte, F.A., Johnston, A., Bruns, N.E., Hochachka, W.M. 2015. Taking a'Big Data' approach to data quality in a citizen science project. Ambio 44: 601-611.

- Lukyanenko, R., Parsons, J., Wiersma, Y.F. 2016. Emerging problems of data quality in citizen science. Conservation Biology 30: 447-449.R Core Team. 2014. "R: A language and environment for statistical computing." From http://www.R-project.org.

- Tulloch, A.I.T., Possingham, H.P., Joseph, L.N., Szabo, J., Martin, T.G. 2013. Realising the full potential of citizen science monitoring programs. Biological Conservation 165: 128-138.

- Turner, M.G., Gardner, R.H, O'neill, R.V. 2001. Landscape ecology in theory and practice. Springer. New York. 